\title{
UPPER AND LOWER BOUNDS OF EIGENVALUES: MODE-CLAMPING THEOREMS*
}

\author{
BY \\ M. SPARKS \\ Science Center, North American Rockwell Corporation, Thousand Oaks, California
}

\begin{abstract}
Upper and lower bounds for the eigenvalues of three types of matrices $M$ are established. If $M$ is written as the sum of a diagonal matrix $D$ plus a matrix $A$, the real parts of the eigenvalues of $M$ must lie between the real parts of the neighboring diagonal elements of $D$, no matter how large the elements of $A$ or how closely spaced the diagonal elements of $D$.

One of the types - that representing one state coupled to possibly all other states of either a quantum-mechanical or classical system-arises in the approximation which gives the golden-rule result that the rate of decay of a state coupled to many other states is linear in time under certain conditions. Another of the types of matrices arises in systems having equal coupling between all states.

1. Introduction. In this paper upper and lower bounds on the eigenvalues of three types of matrices are derived. These matrices arise in many physical problems. For example, they could be the time development matrices for a classical system of coupled oscillators [1], a matrix representation of the quantum mechanical Hamiltonian operator in a discrete basis, etc. The "single-mode-coupling" matrices of Sec. 2 arise [2] in the approximation which gives the golden-rule result that the rate of decay of one state coupled to many other states is linear in time under certain conditions.

The central results are given as three "mode-clamping theorems." In all three cases a matrix $M$ can be written as the sum of a diagonal matrix $D$ plus a matrix $\alpha A$. For $\alpha=0$ the eigenvalues $\lambda_{i}$ of $M$ are equal to the diagonal elements $d_{i}$ of $D$. As $\alpha$ is increased from zero to a finite value, the values of the $\lambda_{j}$ change. The theorems indicate that for the matrices considered these changes are severely restricted. No matter how large $\boldsymbol{\alpha}$ and the elements of $A$ and no matter how closely spaced the $d_{i}$, the real parts of the $\lambda_{i}$ cannot be shifted past the neighboring real parts of the "unperturbed eigenvalues" $d_{i}$ (if certain trivial $d_{i}$ are neglected). The results of the first two theorems, which are not restricted to symmetric matrices, reduce to well-known results [3] in the symmetric case.

The mode-clamping theorems presented here are somewhat similar to Rayleigh's theorem ${ }^{1}$ concerning bounds on the shifts in the phonon frequencies of a system when a single mass is changed. But the theorems here are concerned with a direct perturbation of a matrix, while Rayleigh's theorem concerns a perturbation of a weight operator $W$ in the generalized eigenvector equation $L|V\rangle=\lambda W|V\rangle$.
\end{abstract}

*Received September 7, 1968; revised version received February 13, 1969. The author would like to thank Dr. J. H. Wilkinson, F. R. S., for reading the original manuscript and suggesting a number of substantial modifications. He also gratefully acknowledges conversations with Drs. R. E. DeWames, R. P. Futrelle, W. F. Hall, E. A. Kraut, G. W. Lehman, L. Vredevoe, T. Wolfram, J. Zimmerman, and J. Spanier, who also made helpful suggestions for improving the manuscript.

'For a discussion of Rayleigh's theorem along the same line as the present paper, see [4]. 
Other related results, which may be found in Wilkinson's book [3] in Ch. 2, particularly Secs. 13 and 40-48, apply to more general types of matrices than those considered here. While the matrices considered in the present paper are less general, the bounds are much closer than those obtained for the more general matrices. For example, for the Gerschgorin circle limit theorems (Ch. 2, Sec. 13 of [3]) the bounds become larger as the perturbation becomes larger, while the bounds are independent of the size of the perturbation for the matrices considered here.

The single-mode-coupling theorems 1 and 2 have been used [1] in discussing the relations between the energy eigenvalues and other frequencies of interest in physical systems and in discussing the Clogston-Suhl-Walker-Anderson approximation [5] of replacing an actual coupling between magnons by a single-mode coupling.

2. Single-mode-coupling theorems. Consider the $N$ by $N$ matrix $D+P$, where $D$ has only real diagonal elements $d_{i}$ and $P$ has only elements in the $l$ th row and $l$ th column:

$$
D+P=\left[\begin{array}{ccccccc}
d_{1} & 0 & \cdots & P_{1 l} & \cdots & 0 & 0 \\
0 & d_{2} & & P_{2 l} & \cdots & 0 & 0 \\
\vdots & \vdots & & \vdots & & \vdots & \vdots \\
P_{l 1} & P_{l 2} & \cdots & d_{l} & \cdots & P_{l, N-1} & P_{l N} \\
\vdots & \vdots & & \vdots & & \vdots & \vdots \\
0 & 0 & & P_{N-1, l} & \cdots & d_{N-1} & 0 \\
0 & 0 & & P_{N l} & \cdots & 0 & d_{N}
\end{array}\right]
$$

This perturbation $P$, which will be called a single-mode-coupling perturbation, arises in physical problems in which one $l$ mode (either a state of quantum-mechanical system or a normal mode of a classical system) is coupled to possibly all others. The coupling is represented by $P$.

If $P_{l j}=0$ (or $P_{k l}=0$ ) then $d_{i}\left(\right.$ or $d_{k}$ ) is an eigenvalue $\lambda$ of $D+P$, as seen by expanding the determinant of $D+P-\lambda I$ (where $I$ is the identity matrix) around the $j$ th column (or $k$ th row). When $P_{l j} P_{l l}=0$, the corresponding eigenvalue $\lambda_{u j}=d_{j}$ of $D+P$ will be called an uncoupled eigenvalue. The eigenvalues of $D+P$ corresponding to $P_{l i} P_{i l} \neq 0$ will be called coupled eigenvalues. In solving for the coupled eigenvalues one can omit all rows and columns for which $P_{l i} P_{i l}=0$ since these merely give the uncoupled eigenvalues. In the remaining $N_{r}$ by $N_{r}$ reduced matrix $D_{r}+P_{r}$, which has no uncoupled eigenvalues, it is assumed that a permutation similarity transformation has been applied so that $d_{1}<d_{2}<\cdots<d_{N_{r}}$. Multiple $d_{i}$ and complex $d_{i}$ are considered in Sec. 4.

Expanding the secular determinant $\operatorname{det}\left|D_{r}+P_{r}-\lambda I\right|=0$ around the $l$ th column gives

$$
\mathrm{II}_{i}\left(d_{i}-\lambda\right)-\sum_{k \neq l} P_{l k} P_{k l} \prod_{j \neq k, l}\left(d_{i}-\lambda\right) .
$$

Dividing by $\Pi_{i \neq l}\left(d_{i}-\lambda\right)$ gives $^{2}$

${ }^{2} \mathrm{Eq} .(2)$ is the Brillouin-Wigner perturbation expansion, which truncates after the first term in the sum for single-mode coupling. 


$$
\lambda-d_{l}=\sum_{j \neq l} \frac{P_{l i} P_{i l}}{\lambda-d_{i}} .
$$

First consider the case of $P_{l j} P_{l l}>0$ for all $j \neq l$. Eq. (2) can be solved for $\lambda$ graphically by plotting the left-hand side of (2) as a heavy dashed line and the right-hand side as a heavy solid line, as sketched in Fig. 1. The solutions correspond to the intersections of these two sets of curves, which are marked with circles in the figure. Fig. 1 was sketched specifically for the matrix

$$
D+P=\left[\begin{array}{cccccccc}
1 & 0 & 10^{3} & 0 & 0 & 0 & 0 & 0 \\
0 & 2 & 10^{4} & 0 & 0 & 0 & 0 & 0 \\
10 & 10^{2} & 3 & 10^{3} & i 10^{2} & 10 & 0 & 10^{2} \\
0 & 0 & 0 & 4 & 0 & 0 & 0 & 0 \\
0 & 0 & -i 10^{4} & 0 & 5 & 0 & 0 & 0 \\
0 & 0 & 10^{4} & 0 & 0 & 5+\epsilon & 0 & 0 \\
0 & 0 & 10^{6} & 0 & 0 & 0 & 7 & 0 \\
0 & 0 & 10^{3} & 0 & 0 & 0 & 0 & 8
\end{array}\right]
$$

where $0<\epsilon<2$. The uncoupled eigenvalues are $\lambda_{u 1}=4$ (since $P_{43}=0$ ) and $\lambda_{u 1}=7$ (since $P_{37}=0$ ). For the reduced matrix $D_{r}+P_{r}, N_{r}=6$ and $l=3$. The general results can be stated as the theorem:

Theorem 1. The coupled eigenvalues of the matrix $D+P^{+}$, where $D$ is defined above and $P^{+}$is a single-mode-coupling matrix satisfying $P_{l j}^{+} P_{i l}^{+} \geq 0$ for all $j$, satisfy the inequalities

$$
\begin{aligned}
\lambda_{1} & <d_{1} \\
d_{i-1} & <\lambda_{i}<d_{i} \quad \text { for } \quad d_{i}<d_{l}
\end{aligned}
$$

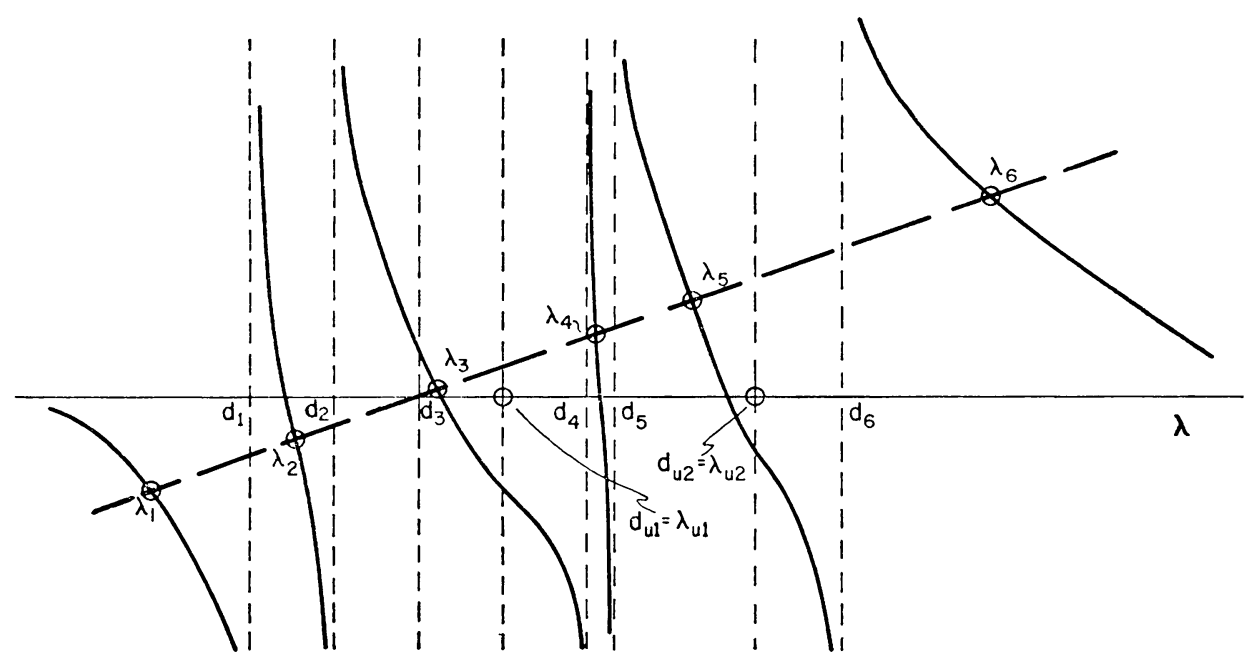

FIg. 1. Construction giving bounds on the eigenvalues $\lambda_{j}$ for nonnegative definite single-mode coupling. Notice the clamping of the eigenvalues between the unperturbed coupled eigenvalues $d_{j}$. 


$$
\begin{aligned}
\lambda_{l-1} & <\lambda_{l}<d_{l+1} \\
d_{i} & <\lambda_{i}<d_{i+1} \text { for } d_{i}>d_{l} \\
\lambda_{N r} & >d_{N}
\end{aligned}
$$

The uncoupled eigenvalues are $\lambda_{u i}=d_{u i}$.

As an example, for the matrix in (3) the relations (4) give

$$
\begin{aligned}
& \lambda_{1}<1 \\
& 1<\lambda_{2}<2 \\
& 2<\lambda_{3}<5 \\
& \lambda_{u 1}=4 \\
& 5<\lambda_{4}<5+\epsilon \quad 5+\epsilon<\lambda_{5}<8 \\
& \lambda_{u 2}=7 \\
& \lambda_{6}>8
\end{aligned}
$$

Next consider the $P$ matrix, denoted by $P^{-}$, whose elements satisfy $P_{{ }_{i j}} P_{i l} \leq 0$ for all $j \neq l$. The right- and left-hand sides of (2) are sketched in Fig. 2 as heavy-dashed and heavy-solid lines, respectively. From this construction it is seen that the coupled eigenvalues of $D+P^{-}$satisfy all of the equations (4) except $\lambda_{\min }\left\langle d_{\min }\right.$ and $\left.\lambda_{\max }\right\rangle d_{\max }$, where min and max denote the minimum and maximum values, respectively, of the coupled eigenvalues excluding $l$. If $d_{\min }<d_{l}<d_{\max }$, as in Fig. 2, the other two coupled eigenvalues are complex. If $d_{l}<d_{\min }$ there are either 0 or 2 eigenvalues of $D+P^{-}$ between $d_{l}$ and $d_{\min }$, as illustrated in Fig. 3. If $d_{l}>d_{\max }$ there are either 0 or 2 eigenvalues of $D+P^{-}$between $d_{\max }$ and $d_{l}$, as illustrated in Fig. 4. These results are summarized as

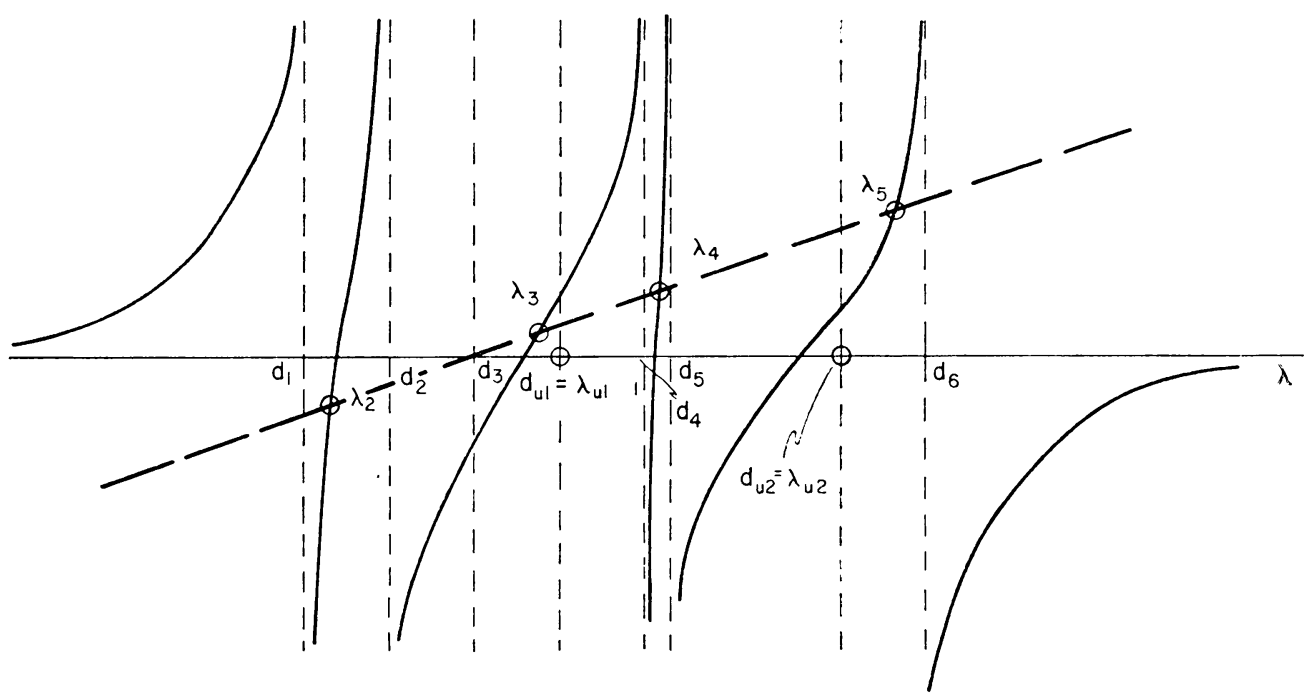

FIG. 2. Construction giving bounds on the eigenvalues $\lambda_{j}$ for nonpositive definite single-mode coupling. Notice the clamping of the eigenvalues between the unperturbed coupled eigenvalues $d_{i}$. 


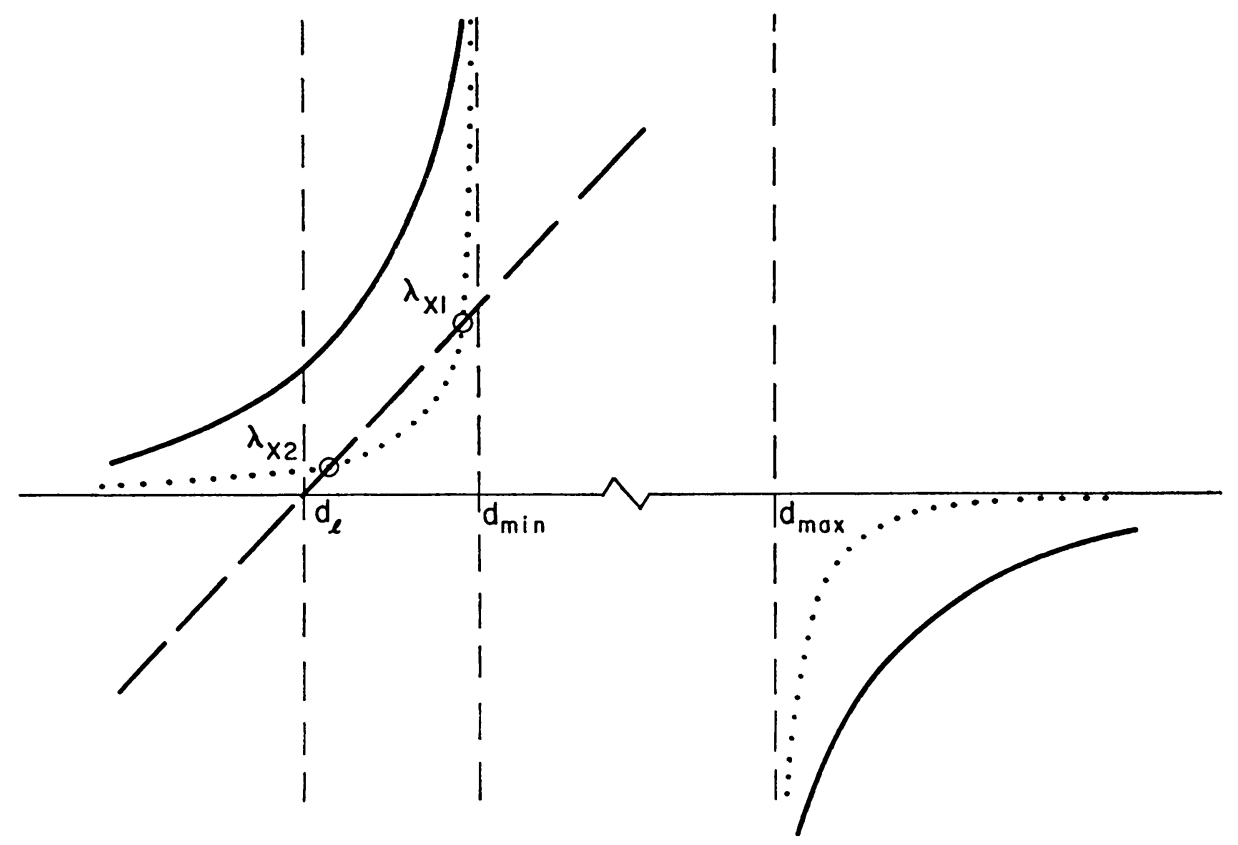

FIG. 3. Clamping of the extra two eigenvalues of $D+P^{-}$when $d_{l}$ lies below the manifold of coupled eigenvalues. The heavy solid curve does not intersect the heavy dashed curve, indicating that the two extra eigenvalues are complex for this "strong coupling" case. The intersections of the heavy dotted curve and the heavy dashed curve give the values of the two extra roots in this "weak coupling" case.

When the two curves touch at one point, the two extra eigenvalues are real and degenerate.

Theorem 2. The coupled eigenvalues of the matrix $D+P^{-}$defined above satisfy all of the equations (4) with the exception of the two eigenvalues for which $\lambda_{\min }<d_{\min }$ and $\lambda_{\max }>d_{\max }$. The remaining two eigenvalues can be real or complex. If they are real, then they are bounded as discussed above. The uncoupled eigenvalue are $\lambda_{u i}=d_{u i}$.

As an example of $d_{l}<d_{\min }$ and $d_{l}>d_{\max }$, consider the matrix

$$
\left[\begin{array}{cc}
1 & a \\
-a^{*} & 2
\end{array}\right]
$$

If we choose $d_{l}=1$ (or $\left.d_{l}=2\right)$, then $d_{\min }=2\left(\right.$ or $\left.d_{\max }=1\right)$. The eigenvalues are

$$
\lambda_{ \pm}=\frac{3}{2} \pm\left[\frac{1}{4}-|a|^{2}\right]^{1 / 2}
$$

For $|a|^{2} \leq \frac{1}{4}$, the two eigenvalues are real. For $|a|^{2}=\frac{1}{4}$, the eigenvalues are real and degenerate $\left(\lambda_{1}=\lambda_{2}=\frac{3}{2}\right)$. For $|a|^{2}>\frac{1}{4}$, the two eigenvalues are complex.

3. Equal-mode-coupling theorem. Now consider the $N$ by $N$ matrix $D+\gamma U$, where $D$ and $I$ have been defined, $U$ is the matrix having all elements equal to one, and 


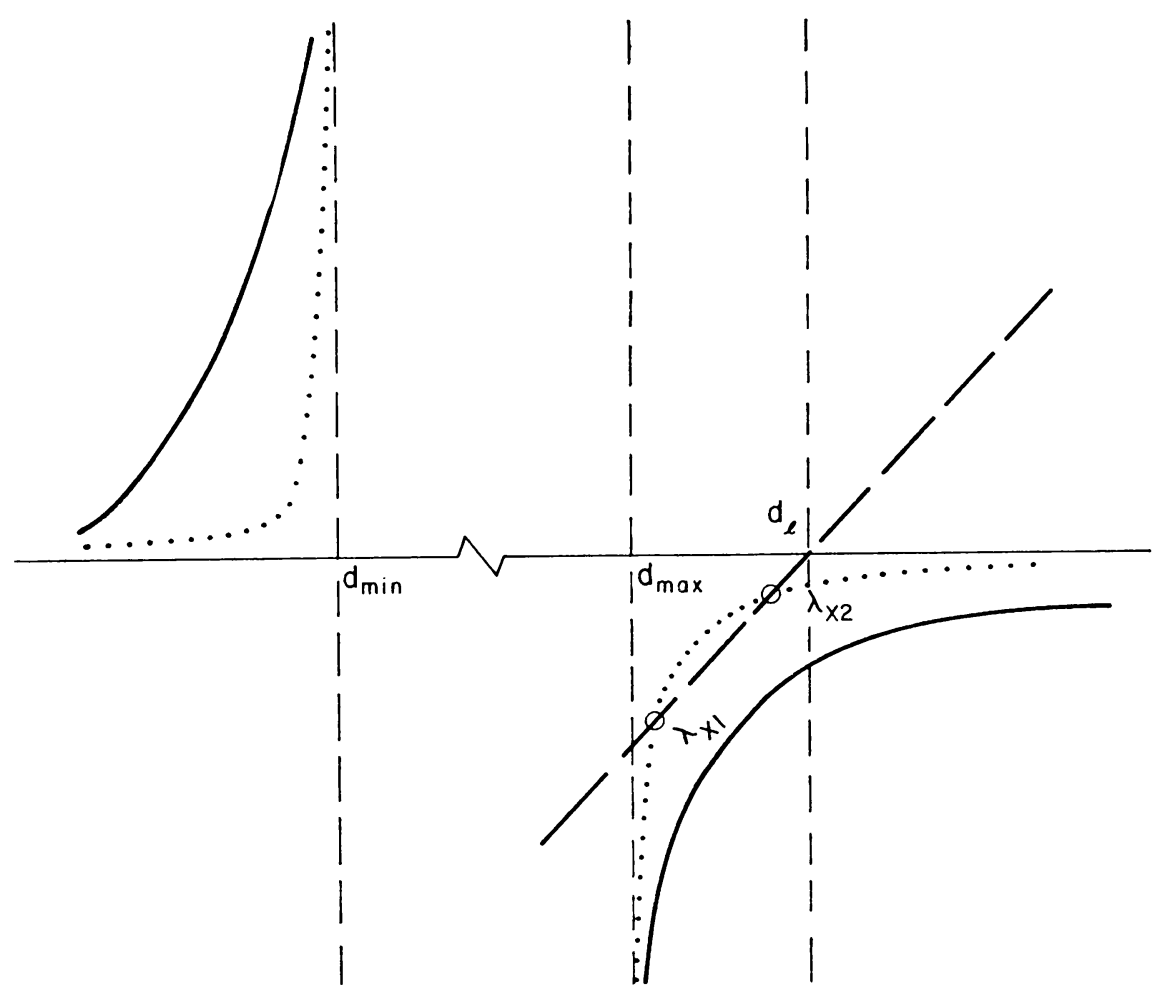

Fig. 4. Clamping of the extra two eigenvalues of $D+P^{-}$when $d_{l}$ lies above the manifold of coupled eigenvalues. The heavy solid curve does not intersect the heavy dashed curve, indicating that the two extra eigenvalues are complex for this "strong coupling" case. The intersection of the heavy dotted curve and the heavy dashed curve give the values of the two extra roots in this "weak coupling" case. When the two curves touch at one point, the two extra eigenvalues are real and degenerate.

$\gamma$ is real:

$$
D+\gamma U=\left[\begin{array}{ccccc}
d_{1}+\gamma & \gamma & \gamma & \cdots & \gamma \\
\gamma & d_{2}+\gamma & \gamma & \cdots & \gamma \\
\gamma & \gamma & d_{3}+\gamma & \cdots & \gamma \\
\vdots & \vdots & \vdots & & \vdots \\
\gamma & \gamma & \gamma & \cdots & d_{N}+\gamma
\end{array}\right]
$$

The perturbation $\gamma U$ will be called an equal-mode-coupling perturbation. As in Sec. 2 , it is assumed without loss of generality that $d_{1}<d_{2}<\cdots<d_{N}$. Expanding $\operatorname{det}|D+\gamma U-\lambda I|$ as the sum of $2^{N}$ determinants gives

$$
\prod_{j}\left(d_{i}-\lambda\right)+\gamma \sum_{j} \prod_{k \neq j}\left(d_{k}-\lambda\right)=0
$$

since all but $N+1$ of the determinants vanish and the remaining $N+1$ are trivial. This gives

$$
\frac{1}{\gamma}=\sum_{i} \frac{1}{\lambda-d_{i}}
$$




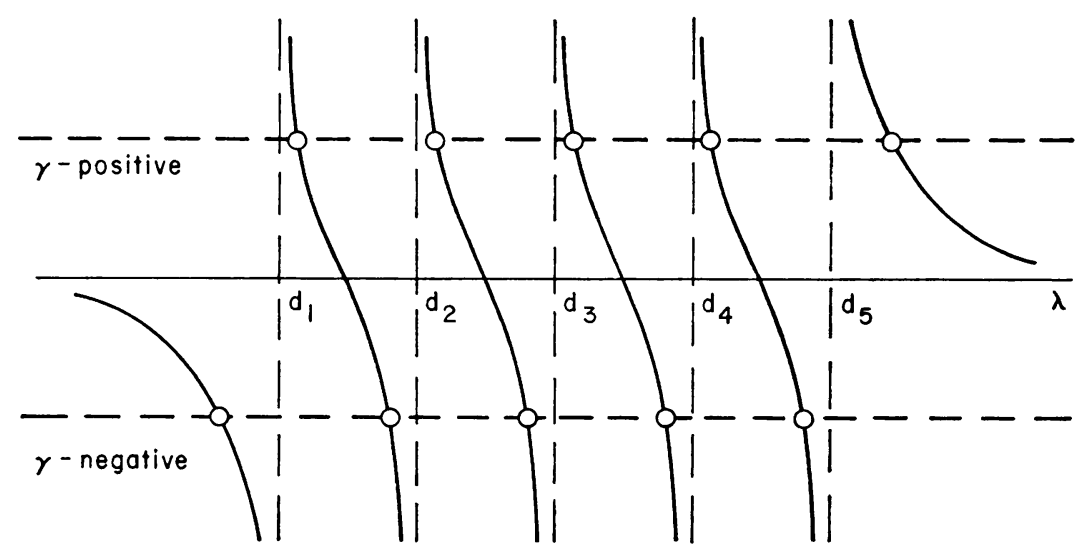

FIG. 5. Construction giving bounds on the eigenvalues $\lambda_{j}$ of $D+\gamma U$ for equal mode coupling. The top set of circles correspond to solutions for $\lambda_{j}$ for the case of $\gamma>0$, and the bottom set for $\gamma<0$.

The final theorem is obtained from the construction in Fig. 5.

Theorem 3. For the $N$ by $N$ matrix $D+\gamma U$ defined above, $N-1$ of the eigenvalues are clamped between the neighboring values of $d_{i}$ :

$$
d_{i}<\lambda_{i}<d_{i+1} \text { for } j=1,2, \cdots N-1 .
$$

For positive $\gamma$ the other eigenvalue satisfies $\lambda_{N}>d_{N}$, and for $\gamma$ negative it satisfies $\lambda_{N}<d_{1}$.

4. Multiple and complex $d_{i}$. The case of multiple $d_{i}$, corresponding to degeneracy in the unperturbed system represented by $D$, is trivial. The characteristic equation ((1) for $D+P$ or (6) for $D+\gamma U$ ) contains the factor $\left(d_{i}-\lambda\right)^{r-1}$ if the multiplicity of $d_{i}\left(\neq d_{l}\right.$ for $\left.P+D\right)$ is $r$; thus the multiplicity of $\lambda=d_{i}$ is $r-1$. For example, in the limit $\epsilon \rightarrow 0$ in (5), it is seen that $\lambda_{4}=d_{4}=d_{5}=5$. For the extreme case of $d_{i}=d_{k} \equiv d$ for all $j$ and $k \neq l$, the value of $r$ is $N-1$; thus $D+P$ has $N-2$ eigenvalues equal to $d$. The other two eigenvalues are easily obtained from (1):

$$
\lambda_{ \pm}=\frac{d+d_{l}}{2} \pm\left[\left(\frac{d-d_{l}}{2}\right)^{2}+\sum_{j \nsim l} P_{l i} P_{i l}\right]^{1 / 2} .
$$

For the extreme degeneracy case of $d_{j}=d$ for all $j$ in $D+\gamma U$, the value of $r$ is $N$; thus there are $N-1$ eigenvalues equal to $d$. From (6) the other eigenvalue is $\lambda_{N}=$ $d+\gamma N$.

Finally, it is obvious in all cases considered that if all $d_{i}$ are replaced by $d_{i}-i \eta$, then all $\lambda$ are replaced by $\lambda-i \eta$. This trivial mathematical fact has the important physical consequence that if all unperturbed modes have equal losses $\eta$, then all of the modes of the coupled system will also have the same loss $\eta$ for single-mode-coupling and for equal-mode-coupling.

\section{ReFERENCES}

[1] M. Sparks (to be published)

[2] L. Schiff, Quantum mechanics, McGraw-Hill, New York, 1955

[3] J. H. Wilkinson, The algebraic eigenvalue problem, Clarendon Press, Oxford, 1965, pp. 84-96.

[4] M. Sparks, Green's functions; state vector approach for physicists (to be published)

[5] A. M. Clogston, H. Suhl, L. R. Walker and P. W. Anderson, J. Phys. Chem. Solids 1, 129 (1956) 